\title{
Toward Quantitative Bright Field TEM Imaging of Ultra Thin Samples
}

Takashi Fujii ${ }^{1}$, Marek Malac ${ }^{2,3}$, Emi Kano $^{2,3}$, Misa Hayashida ${ }^{2}$, Toshie Yaguchi ${ }^{1}$, Ray Egerton ${ }^{3}$

1. Hitachi High-Technologies Corp., Hitachinaka-shi, Ibaraki-ken, Japan.

2. NRC-NANO, Edmonton, Canada.

3. Department of Physics, University of Alberta, Edmonton, Canada.

Two dimensional (2D) materials, such as graphene and transition metal dichalcogenides, are of potential interest from both exploratory research and application perspectives. Here we discuss the potential of bright field transmission electron microscopy (BFTEM) for quantitative mass thickness mapping of ultra thin samples illustrated using graphene $[1,2]$.

Mass thickness measurements of samples with $10 \mathrm{~nm}$ to a few hundred nm thickness are possible with reasonable accuracy by evaluating the image intensity in vacuum $I_{0}$ and the intensity $I_{\text {tr }}$ transmitted through the sample [2] to obtain contrast $C$ :

$$
C=\log \left(I_{0} / I_{\mathrm{tr}}\right)
$$

The results [2] for carbon reproduced in Fig. 1a) show linear dependence of $C$ on mass thickness. Extrapolation to a monolayer-thick graphene films indicates that $C \sim 5 \times 10^{-4}$ to $7 \times 10^{-3}$ can be expected. The measured values of monolayer graphene contrast, collected with small collection angles, in Fig $1 b$ ) are in order of magnitude agreement with the extrapolation from amorphous carbon data.

Clean graphene presents a test sample with well known mass density $\left(\sim 7.7 \times 10^{-2} \mathrm{ug} / \mathrm{cm}^{2}\right)$ and area density of carbon atoms $\left(n=38\right.$ atoms $\left./ \mathrm{nm}^{2}\right)$. The knowledge of mass density and area atomic density makes it possible to compare the measured values of contrast to a simulation. Fig 2 a shows the angular dependence of contrast based on Lenz formula [3] and the relative contribution of the elastic and inelastic scattering to the contrast. The contrast in Fig 2 was estimated using the fact that the transmitted intensity is

$$
\begin{aligned}
& I_{\text {tr }}=I_{0} \exp \left(-\sigma_{\text {tot }} n\right) \\
& C=\sigma_{\text {tot }} n
\end{aligned}
$$

Here $\sigma_{\text {tot }}$ is the total (elastic + inelastic) cross section in $\mathrm{nm}^{2}$ for an electron to be intercepted by an objective aperture, i.e. integrated from objective aperture opening angle $\beta$ to $\pi[2,3]$. Fig $2 \mathrm{a}$ indicates that the contrast increases as the collection angle decreases [2]. Furthermore, below $\sim 3$ mrad angle limiting aperture at $300 \mathrm{kV}$, the contrast arises primarily from inelastic electron scattering while above about 3 mrad the contrast primarily originates from elastic scattering. Fig. $2 b$ shows that the contrast improves by a factor 4 when incident energy decreases from $300 \mathrm{keV}$ to $30 \mathrm{keV}$.

The data presented here were collected in a Hitachi HF-3300 TEM/STEM with cold field emission gun Gatan Ultrascan 1000 camera and pixel size $(\sim 1.5 \mathrm{~nm})^{2}$. To perform measurements with collection angle below $\sim 1$ mrad the objective lens was turned off and the selected area aperture was used as an angle limiting aperture. The sample was held at $400^{\circ} \mathrm{C}$ for several days prior the experiment at a residual atmosphere pressure less than $5 \times 10^{-8}$ torr to prevent contamination. In practice, the non-uniformity of camera gain reference and presence of phase contrast can pose a significant challenge for contrast quantification in ultra thin samples.

In summary, it appears that quantitative mass thickness measurements of an atomic layer thick films may be possible using standard BFTEM. 


\section{References:}

[1] K. Cui et al, Microscopy research and technique 80(8), p. 823.

[2] H-R. Zhang et al, Micron 43(1), p. 8.

[3] R. F. Egerton, EELS in the TEM $3^{\text {rd }}$ ed., Springer Verlag, 2011.
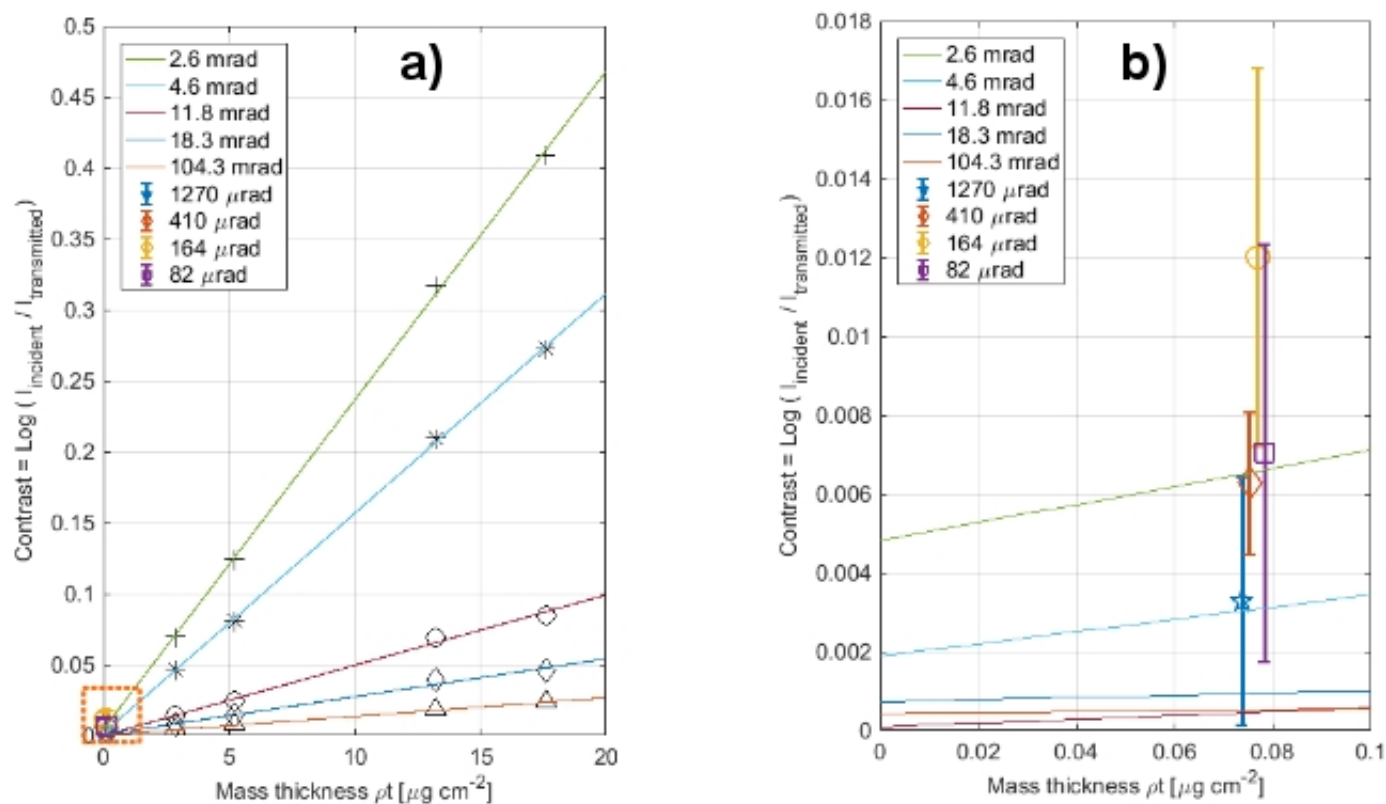

Figure 1. a) Contrast dependence on mass thickness for amorphous carbon film imaged at $300 \mathrm{kV}$. b) The low mass thickness region marked with a rectangle in a) shows the corresponding contrast obtained from single layer graphene. The mass thickness values of graphene were slightly offset in horizontal direction for clarity. The amorphous carbon data were reproduced from [1].
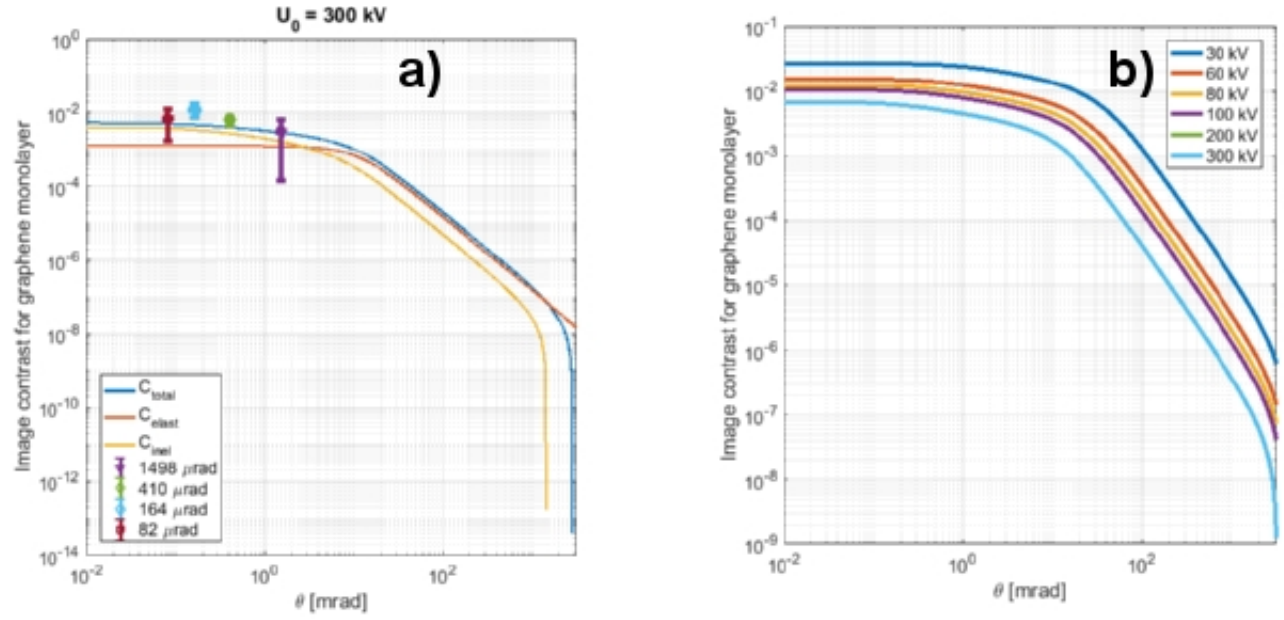

Figure 2. a) Solid lines are the angular dependence of contrast for a single layer graphene estimated using Lenz formula for total contrast (blue), the elastic (red) and inelastic (yellow) contributions. The contrast values measured for a single layer graphene at several collection angles are indicated. b) Angular dependence of total contrast for selected incident electron energies between $30 \mathrm{kV}$ (top, dark blue) and $300 \mathrm{kV}$ (bottom, light blue). 